\title{
Cytokine and neuropeptide levels are associated with pain relief in patients with chronically painful total knee arthroplasty: a pilot study
}

Jasvinder A. Singh ${ }^{1,2,3,4^{*}}$, Siamak Noorbaloochi ${ }^{6,7}$ and Keith L. Knutson ${ }^{5}$

\begin{abstract}
Background: There are few studies with an assessment of the levels of cytokines or neuropeptides as correlates of pain and pain relief in patients with painful joint diseases. Our objective was to assess whether improvements from baseline to 2-months in serum cytokine, chemokine and substance P levels were associated with clinically meaningful pain relief at 2-months post-injection in patients with painful total knee arthroplasty (TKA).

Methods: Using data from randomized trial of 60 TKAs, we assessed the association of change in cytokine/ chemokine/Substance P levels with primary study outcome, clinically important improvement in Western Ontario McMaster Osteoarthritis Index (WOMAC) pain subscale at 2-months post-injection using Student's t-tests and Spearman's correlation coefficient (non-parametric). Patients were categorized as pain responders (20-point reduction or more on 0-100 WOMAC pain) vs. pain non-responders. Sensitivity analysis used 0-10 daytime pain numeric rating scale (NRS) instead of WOMAC pain subscale.

Results: In a pilot study, compared to non-responders $(n=23)$ on WOMAC pain scale at 2-months, pain responders $(n=12)$ had significantly greater increase in serum levels of IL-7, IL-10, IL-12, eotaxin, interferon gamma and TNF-a from baseline to 2 -months post-injection ( $p<0.05$ for all). Change in several cytokine/chemokine and substance $\mathrm{P}$ levels from pre-injection to 2-month follow-up correlated significantly with change in WOMAC pain with correlation coefficients ranging -0.37 to -0.51 : IL-2, IL-7, IL-8, IL-9, IL-16, IL-12p, GCSF, IFN gamma, IP-10, MCP, MIP1b, TNF- $a$ and VEGF $(n=35)$. Sensitivity analysis showed that substance P decreased significantly more from baseline to 2months in the pain responders $(0.54 \pm 0.53 ; n=10)$ than in the pain non-responders $(0.48 \pm 1.18 ; n=9 ; p=0.023)$ and that this change in serum substance $P$ correlated significantly with change in daytime NRS pain, correlation coefficient was $0.53(p=0.021 ; n=19)$. Findings should be interpreted with caution, since cytokine analyses were performed for a sub-group of the entire trial population.
\end{abstract}

Conclusion: Serum cytokine, chemokine and Substance P levels correlated with pain response in patients with painful TKA after an intra-articular injection in a randomized trial.

Keywords: Cytokine, Substance P, Pain, Primary total knee arthroplasty, TKA

\footnotetext{
* Correspondence: Jasvinder.md@gmail.com

${ }^{1}$ Medicine Service, VA Medical Center, 700 19th St S, Birmingham, AL 35233,

USA

${ }^{2}$ Department of Medicine at School of Medicine, University of Alabama at Birmingham Faculty Office Tower, 805B, 510 20th Street S, Birmingham, AL 35294, USA

Full list of author information is available at the end of the article
} International License (http://creativecommons.org/licenses/by/4.0/), which permits unrestricted use, distribution, and reproduction in any medium, provided you give appropriate credit to the original author(s) and the source, provide a link to the Creative Commons license, and indicate if changes were made. The Creative Commons Public Domain Dedication waiver (http://creativecommons.org/publicdomain/zero/1.0/) applies to the data made available in this article, unless otherwise stated. 


\section{Background}

Total knee arthroplasty (TKA) is one of the most successful procedures performed in patients with end-stage osteoarthritis (OA) and other arthritides with refractory pain [1]. While most patients report improved pain and function outcomes after TKA, persistent pain is reported by $6.5 \%$ or more patients post-TKA [2].

The role that cytokines and neurotransmitters play in joint pain is of great interest and has been the focus of recent research [3]. In an animal model of OA, cytokine levels were higher in OA joints, which were reduced by treatment with coenzyme Q-10 that also led to significant pain relief [4]. TNF- $\alpha$ levels were associated with WOMAC pain scores in patients with OA [5]. Joint fluid substance P (SP) levels in knee joints were elevated in painful knee joints with $\mathrm{OA}$ [6] and in preoperative joint fluid in those with greater pain relief after knee arthroplasty [6]. Studies showed that higher levels of cytokines such as interleukin-8 (IL-8), IL-6 and tumor necrosis factor-alpha (TNF- $\alpha$ ) were associated with implant loosening in patients with joint arthroplasty $[7,8]$. To our knowledge, there are limited or no data regarding the role of cytokines in pain severity in patients with painful TKA or relief with interventions.

We examined the data from a randomized trial of patients with persistently painful TKA [9] to test the hypotheses whether in patients with painful TKA, change (baseline to 2-months) in serum cytokine, chemokine and substance P (neuropeptide) levels was associated with clinically meaningful pain relief at 2-months post-injection. We also assessed whether baseline to 2-month change in cytokine/chemokine/substance $\mathrm{P}$ level correlated with change in pain severity from baseline to 2 months.

\section{Methods}

\section{Clinical trial population}

We used the data from patients enrolled in a previously published 6-month, 2-arm, parallel group, blinded placebocontrolled randomized trial (NCT00403273), details provided elsewhere [9]. Briefly, 60 TKAs were randomized in 1:1 to single intra-articular injection of active (onabotulinum toxin A) vs. placebo (saline) to assess its short-term anti-nociceptive efficacy. The research pharmacist prepared computerized randomization with permuted blocks of four patients each and prepared the treatment and placebo syringes using a strict standardized protocol. Both placebo and onabotulinum toxin A injections were transparent and could not be differentiated. In this triple-blind study, patients, investigators (PI, blinded investigators performing assessments, research associates) and the statistician were blinded (all analyses were completed and tables finalized before the pharmacist revealed the designation for group 1 vs. group 2). The PI injected the affected TKA joint using the standardized medial or lateral approach $[10,11]$. This study was adequately powered with 19 patients/group were needed for 80\% power and 24 patients/group for $90 \%$ power (assuming 25\% loss to follow-up), to detect a difference of $43 \%$ in proportion of patients reporting a clinically meaningful improvement in pain, based on previously published studies $[12,13]$.

Primary outcome assessment was at 2-months. A protocol modification to collect blood specimens to understand the mechanism of pain relief in patients with persistently painful TKA was made around the mid-point of patient recruitment upon receipt of federal funds, which provided resources to perform these assays, which were not available previously [9]. The Institutional Review Board (IRB) at the Minneapolis VA approved the study.

\section{Serum cytokine and neuropeptide assays}

Specimens were drawn both at baseline pre-injection and 2-month post-injection. We performed the serum cytokine assay using the BioRad human 27-plex cytokine panel (Cat \# 171-A11127, Bio-Rad, San Diego CA; Table 1; http://www.bio-rad.com/en-jp/sku/m500kcaf0ybio-plex-pro-human-cytokine-27-plex-assay). $100 \mu \mathrm{l}$ of Bio-Plex assay buffer was added to each well of a Multiscreen MABVN $1.2 \mu \mathrm{m}$ microfiltration plate followed by the addition of $50 \mu \mathrm{l}$ of the multiplex bead preparation. Following washing of the beads with the addition of $100 \mu \mathrm{l}$ of wash buffer, $50 \mu \mathrm{l}$ of the samples or the standards was added to each well and incubated with shaking for $30 \mathrm{~min}$ at room temperature. Standard curves were generated with a mixture of 27 cytokine standards and eight serial dilutions ranging from 0 to 32,000 picogram/ $\mathrm{ml}$. The plate was then washed three times followed by incubation of each well in $25 \mu$ l of pre-mixed detection antibodies for $30 \mathrm{~min}$ with shaking. The plate was further washed and $50 \mu \mathrm{l}$ of streptavidin solution were added to each well and incubated for $10 \mathrm{~min}$ at room temperature with shaking. The beads were given a final washing and re-suspended in $125 \mu \mathrm{l}$ of Bio-Plex assay buffer. Cytokine

Table 1 Components of the 27-cytokine panel

Interleukins (IL): IL-1 beta, IL-1 alpha, IL-2, IL-4, IL-5, IL-6, IL-7, IL-8, IL-9, IL10, IL-12 (p70), IL-13, IL-15, IL-17

Basic fibroblast growth factor (FGF)

Eotaxin

granulocyte colony stimulating factor (G-CSF), granulocyte macrophage colony stimulating factor (GM-CSF)

Interferon gamma (IFN- $\gamma$ ), Interferon gamma-induced protein 10 (IP-10)

Monocyte chemoattractant protein-1 (MCP-1)

Macrophage Inflammatory Protein-1 alpha (MIP-1a), MIP-1 beta (MIP-1 $\beta$ )

Platelet-derived growth factor (PDGF)

Regulated upon Activation, Normal T cell Expressed and presumably Secreted (RANTES)

Tumor necrosis factor-alpha (TNF-a)

Vascular endothelial growth factor (VEGF) 
levels in the sera were quantitated by analyzing $100 \mu \mathrm{l}$ of each well on a Bio-Plex using Bio-Plex Manager software version 4.0.

Serum neuropeptide assay included enzyme linked immunoassays for Substance P (SP), (R\&D Systems, Minneapolis, MN; https://www.rndsystems.com/products/ substance-p-parameter-assay-kit_kge007\#product-details), which was performed as per manufacturer's instructions in a standard fashion. Samples, standards and controls were placed in a 96 well plate pre-coated with a polyclonal antibody. Primary antibody (for SP) solution and SP conjugated to horseradish peroxidase, were added to the wells and the plate was incubated overnight at $4^{\circ}$ Celsius. After washing, substrate solution was added to the wells, incubated for $30 \mathrm{~min}$, at room temp, in the dark and stop solution was added. The optical density of wells was determined using a micro plate reader set to $450 \mathrm{~nm}$, with a reference wavelength of $570 \mathrm{~nm}$.

\section{Statistical analyses}

Change in serum cytokine, chemokine and Substance P levels were calculated as baseline minus 2-month levels for each patient, where specimen was available. We decided a priori not to impute any values. We defined clinically important improvement in pain as the following: WOMAC pain scale as 20-point absolute improvement or more (range $0-100$; higher = more pain; main analysis); or 2-point reduction or more in index TKA daytime numeric rating scale (NRS) pain severity on $0-10$ scale (higher = more pain) at 2-month post-injection (sensitivity analysis), as previously published [14]. Patients were categorized as pain responder (achieved clinically important improvement in WOMAC pain) vs. pain non-responders (did not achieve this clinically improvement in WOMAC pain). We used student's t-tests to compare the mean change in cytokine chemokine and Substance P levels baseline to 2 -months post-injection between pain responders and non-responders. We assessed non-parametric Spearman's correlation between cytokine/neurotransmitter level change from baseline to 2-months and the decrease in WOMAC pain (main analysis) or 0-10 daytime pain numeric rating scale (NRS; sensitivity analyses), baseline to 2-months, to account for non-normal distributions.

\section{Results}

Patients in the randomized trial had a mean age of 67 years, 84\% were male and 96\% were Caucasian. The mean duration of TKA pain was 4.5 years (SD, 4.8) and $75 \%$ were primary TKAs.

Compared to non-responders $(n=23)$ on WOMAC pain scale at 2-months, pain responders $(n=12)$ had significantly greater increase in serum levels of IL-7, IL-10, IL-12 (p70), eotaxin, IFN- $\gamma$ and TNF- $\alpha$ from baseline to 2months post-injection $(p<0.05$ for all; Table 2$)$. Several other cytokines showed a non-significant trend by painresponder status $(p \leq 0.32$; Table 2$)$. In sensitivity analysis in a smaller set of patients, who reported daytime NRS pain on $0-10$ scale, serum substance $P$ decreased significantly more in the pain responders $(0.54 \pm 0.53$; $n=10)$ than in the pain non-responders $(0.48 \pm 1.18 ; n=9$; $p=0.023)$ 2-months post-injection.

Change in several cytokine and chemokine levels from pre-injection to 2-month follow-up correlated significantly with change in WOMAC pain with correlation coefficients ranging -0.37 to -0.51 : IL-2, IL-7, IL-8, IL-9, IL-16, IL-12 (p70), GCSF, IFN- $\gamma$, IP-10, MCP, MIP1b, TNF- $\alpha$ and VEGF ( $n=35$; Table 3$)$. In sensitivity analysis, we additionally noted a change in serum substance $P$ from pre-injection to 2-month follow-up correlated significantly with change in daytime NRS pain, correlation coefficient was $0.53(p=0.021 ; n=19)$.

\section{Discussion}

In this ancillary pilot study, we assessed whether changes in serum levels of cytokines, chemokines and Substance $\mathrm{P}$ from baseline to 2-month post-injection were associated with clinically meaningful pain relief at 2-month post-injection. We performed a mechanistic study and used data from our randomized study of intra-articular injection for painful TKA [9]. In many patients, the pain improvement lasted through the 6-month follow-up period, indicating that the joint pain relief was somewhat durable [9]. We found that several cytokine levels, including IL-7, IL-10, IL-12 (p70), eotaxin, IFN- $\gamma$ and TNF- $\alpha$, changed significantly more in WOMAC pain responders compared to pain non-responders (responders defined as those with pain decrement of 20 points or more on $0-100$ scale). Correlation analyses identified additional cytokines with moderate correlations with WOMAC pain scores (baseline to 2-month change in cytokine level with baseline to 2-month change in WOMAC pain) in addition to these, including IL-2, IL-8, IL-9, IL-16, GCSF, IP-10, MCP, MIP1b and VEGF. In sensitivity analysis using a smaller dataset, serum substance $P$ level reduction was greater in pain responders using the 0-10 daytime NRS pain than pain non-responders (pain decrement of two points or more o $0-10$ scale). The direction and magnitude were similar to the responder analysis by WOMAC pain, however, standard deviations were larger in WOMAC pain analysis, leading to the difference in substance $\mathrm{P}$ levels being non-significant $(p=0.32)$ in the main analysis, but significant in sensitivity analysis $(p=0.023)$. This is a hypothesis-generating study and therefore, these findings need to be replicated in future studies.

The traditional view of cytokines/chemokines being either anti- or pro-inflammatory [15] has been challenged [16], since they can serve either role depending on the condition and the body organ. Higher levels of pro- 
Table 2 Association of change in serum cytokine and neurotransmitter levels from baseline to 2-months with pain responder status on WOMAC pain at 2-month post-injection in painful TKA

\begin{tabular}{|c|c|c|c|c|c|c|}
\hline & WOMAC Pain Responder* & $\mathrm{N}$ & $\begin{array}{l}\text { Mean Change } \\
\text { (FU-Baseline) }\end{array}$ & Std. Deviation & Std. Error Mean & $P$-value \\
\hline \multirow[t]{2}{*}{ Interleukin (IL)-7 } & No & 23 & 0.084 & 0.91 & 0.19 & 0.01 \\
\hline & Yes & 12 & 1.07 & 1.17 & 0.34 & \\
\hline \multirow[t]{2}{*}{ IL-10 } & No & 23 & 8.51 & 20.90 & 4.36 & 0.01 \\
\hline & Yes & 12 & 27.72 & 21.56 & 6.22 & \\
\hline \multirow[t]{2}{*}{ IL-12 p70 } & No & 23 & 3.36 & 8.17 & 1.70 & 0.004 \\
\hline & Yes & 12 & 12.91 & 9.60 & 2.77 & \\
\hline \multirow[t]{2}{*}{ Eotaxin } & No & 23 & -2.03 & 13.92 & 2.90 & 0.046 \\
\hline & Yes & 12 & 7.85 & 12.28 & 3.54 & \\
\hline \multirow[t]{2}{*}{ Interferon gamma (IFN- $\gamma)$} & No & 23 & -1.24 & 23.18 & 4.83 & 0.03 \\
\hline & Yes & 12 & 15.61 & 13.35 & 3.85 & \\
\hline \multirow[t]{2}{*}{ Tumor necrosis factor-alpha (TNF-a) } & No & 23 & 4.46 & 21.32 & 4.44 & 0.03 \\
\hline & Yes & 12 & 22.22 & 24.65 & 7.12 & \\
\hline \multirow[t]{2}{*}{$\mid \mathrm{L}-4$} & No & 23 & -0.02 & 0.16 & 0.03 & 0.12 \\
\hline & Yes & 12 & 0.06 & 0.12 & 0.03 & \\
\hline \multirow[t]{2}{*}{ IL-6 } & No & 23 & -15.90 & 275.66 & 57.48 & 0.09 \\
\hline & Yes & 12 & 137.35 & 182.09 & 52.56 & \\
\hline \multirow[t]{2}{*}{ IL-13 } & No & 23 & 4.90 & 13.25 & 2.76 & 0.15 \\
\hline & Yes & 12 & 12.18 & 14.87 & 4.29 & \\
\hline \multirow[t]{2}{*}{ IL-15 } & No & 23 & 0.31 & 11.87 & 2.47 & 0.09 \\
\hline & Yes & 12 & 7.36 & 10.52 & 3.038 & \\
\hline \multirow[t]{2}{*}{ Macrophage Inflammatory Protein-1 beta (mip1b) } & No & 23 & 5.55 & 91.43 & 19.06 & 0.16 \\
\hline & Yes & 12 & 53.35 & 96.39 & 27.82 & \\
\hline \multirow[t]{2}{*}{ Substance P } & No & 14 & 0.08 & 1.11 & 0.298 & 0.32 \\
\hline & Yes & 5 & -0.46 & 0.61 & 0.273 & \\
\hline
\end{tabular}

Only those associations that either had a significant $p$-value or a $p$-value $<0.33$ are listed; The levels of other cytokine did not differ significantly between pain responders and pain non-responders (IL-1 beta, IL-1 alpha, IL-2, IL-5, IL-8, IL-9, IL-17, Basic FGF, G-CSF, GM-CSF, IP-10, MCP-1, MIP-1 alpha, PDGF, RANTES, and VEGF). Significant $p$-values $<0.05$ are in bold

Positive changes mean that at the 2-month follow-up time, the levels were higher than the baseline and a negative sign means follow-up levels were lower WOMAC Pain Responder* is defined as reduction in WOMAC pain subscale of 20 or more $0-100$ scale

inflammatory cytokines have been linked to pain [17-20]. On the other hand, change in pro- and anti-inflammatory cytokines with treatment in other pain conditions do not map precisely to their associations with pain condition at baseline [21-24]. Studies that have investigated the potential mechanisms of joint pain relief in intervention studies are lacking. Such studies can provide insights into mechanisms of action of an intervention, and discover mediators of joint pain relief in patients with OA. Our study begins to fill this knowledge gap by providing data among patients with painful joint arthroplasty.

Other recent uncontrolled studies have documented a potential role of cytokines, chemokines and Substance $\mathrm{P}$ in the failure of total joint replacement. In a recent study, both IL-1 beta and IL-2 levels were significantly lower $(p<0.025)$ in 10 patients with stable, painless, well-functioning, cemented total knee or hip arthroplasties (TKA/THA) than patients with aseptically loosened, painful, arthroplasties [25]. Genetic variants of pro-inflammatory cytokines TNF-alpha and IL6 were associated with susceptibility to severe osteolysis after THA, a condition that is associated with increasing pain and functional limitation [7]. Compared to OA, aseptic loosening of TKA was associated with up-regulated expression of several cytokines including IL-8 and MMP9 and low levels of inflammatory cytokines [8].

In patients undergoing revision surgery for painful primary hip arthroplasty, nerve fibers with positive immunostaining to Substance P were found in bone-prosthesis interface membranes [26]. Joint fluid Substance P levels were elevated in painful knee joints with osteoarthritis that underwent TKA, but not in normal/asymptomatic contralateral knees [6]. Significantly greater pain relief after knee arthroplasty was seen in patients with an 
Table 3 Non-parametric correlation of change in serum cytokine and neurotransmitter levels from baseline to 2-months with change in WOMAC pain at 2-month post-injection in painful TKA, showing statistically significant associations

\begin{tabular}{llc}
\hline $\begin{array}{l}\text { Baseline to 2-month change } \\
\text { in serum levels* }\end{array}$ & $\begin{array}{l}\text { Spearman's correlation } \\
\text { coefficient }\end{array}$ & $\begin{array}{l}P \text {-value } \\
\text { IL2 }\end{array}$ \\
IL7 & -0.37 & 0.03 \\
IL8 & -0.42 & 0.01 \\
IL9 & -0.51 & $\mathbf{0 . 0 0 2}$ \\
IL16 & -0.50 & $\mathbf{0 . 0 0 2}$ \\
IL12p70 & -0.48 & $\mathbf{0 . 0 0 4}$ \\
GCSF & -0.56 & $<\mathbf{0 . 0 0 1}$ \\
IFN gamma & -0.34 & 0.04 \\
IP10 & -0.48 & $\mathbf{0 . 0 0 3}$ \\
MCP & -0.38 & 0.02 \\
MIP1b & -0.35 & 0.03 \\
TNF-alpha & -0.49 & $\mathbf{0 . 0 0 3}$ \\
VEGF & -0.42 & 0.01 \\
\hline
\end{tabular}

*Data from 35 patients were available

Only those cytokines that had a significant $p$-value $<0.05$ are listed in bold; other cytokines were not significantly associated with change in WOMAC pain at 2-month post-TKA

elevated preoperative joint fluid Substance P level compared to patients with normal Substance P levels [6]. In an animal model of OA, MMP-13, IL-1b, IL-6 and IL-15 were up-regulated in $\mathrm{OA}$ joints and the levels were reduced by treatment with coenzyme Q-10 that led to significant pain relief [4]. In a study of 47 OA patients, TNFalpha levels were associated with WOMAC pain and overall scores [5]. Thus, cytokine levels have been correlated with OA and arthroplasty joint pain in observational studies. Our study provides evidence showing that a change in cytokine levels correlated with pain relief in patients with painful TKA in a clinical trial who had a clinically meaningful pain relief after an intra-articular injection.

Our study findings must be interpreted considering study limitations. The sample size for this ancillary study was small. A protocol modification was made to the main study on receipt of federal funding that allowed us to collect specimens and perform analyses, and therefore these analyses were performed on a subset of the main trial, since almost $40 \%$ of the study cohort had already been enrolled in the study. Therefore, we likely missed several important findings due to type II error. Subsequent studies should enroll a larger number of patients to explore this important aspect of treatment for patients with painful arthroplasty. We examined the patients in the pain responder vs. non-responder categories, as specified a priori in our analytic plan. Since our hypothesis was to assess the cytokine, chemokine and neuropeptide mediators of joint pain relief in painful arthroplasty, we limited our analyses by whether patient was or was not a responder by clinically meaningful pain relief at 2-months postinjection. We adopted this approach rather than comparing Botulinum toxin group vs. placebo group, since we only had samples on half of the study participants in the RCT and both groups of patients with samples consisted of a mix of pain responders and non-responders, leading to an inadequate sample size for this comparison. We believe that the associations we noted are unlikely to be drug-specific, since responders included patients from both intervention and control arms. This analysis addressed our main objective to better understand the mechanism of pain improvement in patients who have had a failed TKA, and are undergoing a medical treatment to improve pain. Study strengths include the randomized, blinded study design, use of paired samples and focus on a condition that has significant public health impact. The presence of significant associations, even under the stringent categorization (responders/non-responders), with the current sample size, supports our underlying theoretical framework that cytokine, chemokine and substance P level changes after an intra-articular injection might be associated with pain relief.

\section{Conclusions}

In conclusion, our exploratory study shows that patients who experience pain relief after an intra-articular injection have a different pattern of change in serum cytokine/chemokine levels than patients without pain relief. Our study identifies several cytokines that might play a role in relief of joint pain with local therapies. Our study has generated interesting hypotheses of mechanisms of pain relief in patients with painful TKA. Future studies need to confirm our findings in patients with painful arthroplasty, with similar intra-articular interventions, but also other dissimilar interventions, since pain pathways/mechanisms may be similar.

\section{Abbreviations}

NRS: Numeric rating scale; OA: Osteoarthritis; TKA: Total knee arthroplasty; WOMAC: Western Ontario and McMaster Universities Osteoarthritis Index

\section{Acknowledgements}

None.

\section{Funding}

This study was supported by the resources and use of facilities at Birmingham and Minneapolis Veterans Affairs medical centers and the NIH CTSA award 1 KL2 RR024151-01 (Mayo Clinic Center for Clinical and Translational Research). JAS is supported by the resources and the use of facilities at the VA Medical Center at Birmingham, Alabama, USA.

\section{Availability of data and materials}

We are ready to share these data with colleagues after appropriate institutional, ethics and patient privacy requirements have been met.

\section{Authors' contributions}

JAS designed the study, developed study protocol, collected data, reviewed data analyses and wrote the first draft of the paper. SN performed the data analyses. KK supervised all cytokine and other assays. All authors revised the manuscript, read, and approved the final manuscript. 


\section{Authors' information}

JAS is the co-chair of the special Interest group assessing the outcome measures in arthroplasty and has served as expert/lead on task forces for the specialty societies and the US FDA. Other authors have no other relevant information

Each author certifies that his or her institution has approved the human protocol for this investigation and that all investigations were conducted in conformity with ethical principles of research.

"The views expressed in this article are those of the authors and do not necessarily reflect the position or policy of the Department of Veterans Affairs or the United States government."

\section{Competing interests}

JAS has received research grants from Takeda and Savient and consultant fees from Savient, Takeda, Regeneron, Merz, Bioiberica, Crealta and Allergan pharmaceuticals, WebMD, UBM LLC and the American College of Rheumatology. JAS serves as the principal investigator for an investigator-initiated study funded by Horizon pharmaceuticals through a grant to DINORA, Inc., a 501 (c)(3) entity. JAS is a member of the executive of OMERACT, an organization that develops outcome measures in rheumatology and receives arms-length funding from 36 companies; a member of the American College of Rheumatology's (ACR) Annual Meeting Planning Committee (AMPC); Chair of the ACR Meet-the-Professor, Workshop and Study Group Subcommittee; and a member of the Veterans Affairs Rheumatology Field Advisory Committee. Other authors have no relevant disclosures. None of the authors have any non-financial disclosures.

\section{Ethics approval and consent to participate}

The Institutional Review Board (IRB) at the Minneapolis VA approved the study. A written informed consent was obtained from all patients.

\section{Author details}

${ }^{1}$ Medicine Service, VA Medical Center, 700 19th St S, Birmingham, AL 35233 , USA. ${ }^{2}$ Department of Medicine at School of Medicine, University of Alabama at Birmingham Faculty Office Tower, 805B, 510 20th Street S, Birmingham, AL 35294, USA. ${ }^{3}$ Division of Epidemiology at School of Public Health, University of Alabama at Birmingham, 1720 Second Ave. South, Birmingham, AL 35294-0022, USA. ${ }^{4}$ Department of Orthopedic Surgery, Mayo Clinic College of Medicine, 200 1st St SW, Rochester, MN 55905, USA. ${ }^{5}$ Department of Immunology, Mayo Clinic College of Medicine, 200 1st St SW, Rochester, MN 55905, USA. ${ }^{6}$ Center for chronic disease Outcomes Research, Minneapolis Veterans Affairs Health are System Center, Minneapolis, MN 55121, USA. ${ }^{7}$ Department of Medicine, University of Minnesota, 401 East River Parkway, Minneapolis, MN 55455, USA.

Received: 4 October 2016 Accepted: 21 December 2016 Published online: 14 January 2017

\section{References}

1. Singh JA, Vessely MB, Harmsen WS, Schleck CD, Melton 3rd L, Kurland RL Berry DJ. A population-based study of trends in the use of total hip and total knee arthroplasty, 1969-2008. Mayo Clin Proc. 2010;85(10):898-904.

2. Singh JA, Gabriel S, Lewallen D. The impact of gender, age, and preoperative pain severity on pain after TKA. Clin Orthop Relat Res. 2008; 466(11):2717-23.

3. Miller RE, Miller RJ, Malfait AM. Osteoarthritis joint pain: The cytokine connection. Cytokine. 2014;70(2):185-93.

4. Lee J, Hong YS, Jeong JH, Yang EJ, Jhun JY, Park MK, Jung YO, Min JK, Kim HY, Park SH, et al. Coenzyme Q10 ameliorates pain and cartilage degradation in a rat model of osteoarthritis by regulating nitric oxide and inflammatory cytokines. PLoS One. 2013;8(7):e69362.

5. Orita S, Koshi T, Mitsuka T, Miyagi M, Inoue G, Arai G, Ishikawa T, Hanaoka E, Yamashita K, Yamashita M, et al. Associations between proinflammatory cytokines in the synovial fluid and radiographic grading and pain-related scores in 47 consecutive patients with osteoarthritis of the knee. BMC Musculoskelet Disord. 2011;12:144.

6. Pritchett JW. Substance $P$ level in synovial fluid may predict pain relief after knee replacement. J Bone Joint Surg (Br). 1997;79(1):114-6.

7. Gallo J, Mrazek F, Petrek M. Variation in cytokine genes can contribute to severity of acetabular osteolysis and risk for revision in patients with ABG 1 total hip arthroplasty: a genetic association study. BMC Med Genet. 2009;10:109.
8. Tomankova T, Kriegova E, Fillerova R, Luzna P, Ehrmann J, Gallo J. Comparison of periprosthetic tissues in knee and hip joints: differential expression of CCL3 and DC-STAMP in total knee and hip arthroplasty and similar cytokine profiles in primary knee and hip osteoarthritis. Osteoarthritis Cartilage. 2014;22(11):1851-60.

9. Singh JA, Mahowald ML, Noorbaloochi S. Intraarticular botulinum toxin A for refractory painful total knee arthroplasty: a randomized controlled trial. J Rheumatol. 2010;37(11):2377-86.

10. Courtney P, Doherty M. Joint aspiration and injection. Best Pract Res Clin Rheumatol. 2005;19(3):345-69.

11. Lopes RV, Furtado RN, Parmigiani L, Rosenfeld A, Fernandes AR, Natour J. Accuracy of intra-articular injections in peripheral joints performed blindly in patients with rheumatoid arthritis. Rheumatology (Oxford). 2008;47(12):1792-4.

12. Bellamy N, Bell MJ, Goldsmith CH, Pericak D, Walker V, Raynauld JP, Torrance GW, Tugwell P, Polisson R. The effectiveness of hylan G-F 20 in patients with knee osteoarthritis: an application of two sets of response criteria developed by the OARSI and one set developed by OMERACT-OARSI. Osteoarthritis Cartilage. 2005;13(2):104-10.

13. Singh J, Mahowald M, Krug H, Gioe T, Santos E, Schmidt R. Intra-articular Botulinum Toxin A for Painful Total Knee Arthroplasty (TKA). Arthritis Rheum. 2006;54(9; Supp):S541-542.

14. Farrar JT, Portenoy RK, Berlin JA, Kinman JL, Strom BL. Defining the clinically important difference in pain outcome measures. Pain. 2000;88(3):287-94.

15. Opal SM, DePalo VA. Anti-inflammatory cytokines. Chest. 2000;117(4):1162-72.

16. Cavaillon JM. Pro- versus anti-inflammatory cytokines: myth or reality. Cell Mol Biol (Noisy-le-grand). 2001;47(4):695-702.

17. Alexander RB, Ponniah S, Hasday J, Hebel JR. Elevated levels of proinflammatory cytokines in the semen of patients with chronic prostatitis/ chronic pelvic pain syndrome. Urology. 1998;52(5):744-9.

18. Hu C, Yang H, Zhao Y, Chen X, Dong Y, Li L, Dong Y, Cui J, Zhu T, Zheng P, et al. The role of inflammatory cytokines and ERK1/2 signaling in chronic prostatitis/chronic pelvic pain syndrome with related mental health disorders. Sci Rep. 2016;6:28608.

19. Jiang YH, Peng CH, Liu HT, Kuo HC. Increased pro-inflammatory cytokines, C-reactive protein and nerve growth factor expressions in serum of patients with interstitial cystitis/bladder pain syndrome. PLoS One. 2013;8(10):e76779.

20. Kiguchi N, Kobayashi Y, Kishioka S. Chemokines and cytokines in neuroinflammation leading to neuropathic pain. Curr Opin Pharmacol. 2012;12(1):55-61.

21. Bushehri A, Chow E, Zhang L, Azad A, Vuong S, Pasetka M, Zhou M, Hird A, Dennis K, McDonald R, et al. Urinary cytokines/chemokines pattern in patients with painful bone metastases undergoing external beam radiotherapy experiencing pain flare. Ann Palliat Med. 2016;5(2):107-15.

22. Degenhardt BF, Johnson JC, Fossum C, Andicochea CT, Stuart MK. Changes in Cytokines, Sensory Tests, and Self-Reported Pain Levels After Manual Treatment of Low Back Pain. Clin Spine Surg. 2016. [Epub ahead of print]. doi:10.1097/BSD.0000000000000231.

23. Johnston IN, Milligan ED, Wieseler-Frank J, Frank MG, Zapata V, Campisi J, Langer S, Martin D, Green P, Fleshner M, et al. A role for proinflammatory cytokines and fractalkine in analgesia, tolerance, and subsequent pain facilitation induced by chronic intrathecal morphine. J Neurosci. 2004;24(33):7353-65.

24. Kraychete DC, Sakata RK, Issy AM, Bacellar O, Jesus RS, Carvalho EM. Proinflammatory cytokines in patients with neuropathic pain treated with Tramadol. Rev Bras Anestesiol. 2009;59(3):297-303.

25. Chadha HS, Wooley PH, Sud S, Fitzgerald Jr RH. Cellular proliferation and cytokine responses to polymethylmethacrylate particles in patients with a cemented total joint arthroplasty. Inflamm Res. 1995;44(4):145-51.

26. Ahmed M, Bergstrom J, Lundblad H, Gillespie WJ, Kreicbergs A. Sensory nerves in the interface membrane of aseptic loose hip prostheses. J Bone Joint Surg (Br). 1998;80(1):151-5. 